\title{
HEIGHT OF EXCEPTIONAL CHARACTER DEGREES ${ }^{1}$
}

\author{
DAVID A. SIBLEY
}

\begin{abstract}
Suppose a finite group $G$ has an abelian Sylow $p$-group $P$ which is a T.I. set and whose normalizer is a Frobenius group with kernel $P$. It is shown that the degree of an exceptional character of $G$ is not divisible by $p$. That is, exceptional characters have height 0 in the principal $p$-block of $G$.
\end{abstract}

We assume

\section{$P$ is a Sylow p-group of the finite group $G, P$ is an Abelian}

T.I. set, and $N=N_{G}(P)$ is a Frobenius group with Frobenius kernel $P$.

From the Brauer-Suzuki theory of exceptional characters we find the following facts relating characters of $G$ and $N$ when $G$ has at least two classes of $p$-elements. Let $\lambda_{1}, \ldots, \lambda_{t}$ be the exceptional characters of $N$. There are an integer $c$ and a sign $\delta= \pm 1$ and exceptional characters $\Lambda_{1}, \ldots, \Lambda_{t}$ of $G$ such that $\Lambda_{i}(g)=\delta \lambda_{i}(g)+c$ for all $i=1, \ldots, t$ and all $g \in P^{\#}$. All other irreducible characters of $G$ are constant on $P^{\#}$. We exploit this last fact to show

Theorem. Suppose (*) holds and $G$ has at least two classes of p-elements. Then $p \nmid \Lambda_{i}(1)$ for any $i$.

Recently the author showed that $c=0$ when $G$ has at least three classes of $p$-elements [1]. Since $\Lambda_{i}(1) \equiv \delta s+c(\bmod |P|)$, where $s=|N: P|$, the Theorem follows trivially in this case. Thus, the only new part of the Theorem is the case for which $G$ has exactly two classes of $p$-elements.

We now begin the proof. The values of the $\Lambda_{i}$ on $p$-regular elements of $G$ are independent of $i$, so we will drop the subscript $i$ for such elements. For any $x, y, z \in G$ we define class multiplication constants $\gamma(x, y, z)$ in the usual way. Choose $a, b, c \in P^{\#}$ and $x$ a $p$-regular element of $G$. The usual character formula for class multiplication constants yields

$$
\begin{aligned}
\gamma(a, b, x)-\gamma(a, c, x) & =\frac{|G|}{|P|^{2}} \frac{\Lambda(x)}{\Lambda(1)} \sum_{i}\left(\Lambda_{i}(a) \Lambda_{i}(b)-\Lambda_{i}(a) \Lambda_{i}(c)\right) \\
& =\frac{|G|}{|P|} \frac{\Lambda(x)}{\Lambda(1)}\left(\delta_{a b}-\delta_{a c}\right)
\end{aligned}
$$

Received by the editors February 16, 1976.

AMS (MOS) subject classifications (1970). Primary 20C15; Secondary $20 \mathrm{H} 20$.

${ }^{1}$ This research was supported in part by NSF Grant MPS75-06334. 
by an easy calculation involving column orthogonality. Here $\delta_{g h}$ is defined for any $g, h \in P^{\#}$ via

$$
\delta_{g h}= \begin{cases}1 & \text { if } g \sim h^{-1}, \\ 0 & \text { otherwise. }\end{cases}
$$

Since $G$ has at least two classes of $p$-elements, we can choose $a, b, c \in P^{\#}$ so that $\left(\delta_{a b}-\delta_{a c}\right)=1$. Thus

$$
\gamma(a, b, x)-\gamma(a, c, x)=\frac{|G|}{|P|} \frac{\Lambda(x)}{\Lambda(1)}
$$

is an integer.

Now suppose by way of contradiction that $p \mid \Lambda(1)$. Then also $p \mid \Lambda(x)$ for any $p$-regular element $x$ by the above calculation. We now calculate $\left\|\Lambda_{i}\right\|^{2}$. This gives

$$
1=\left\|\Lambda_{i}\right\|^{2}=\frac{\Lambda(1)^{2}}{|G|}+\sum^{\prime} \frac{\Lambda_{i}(g) \overline{\Lambda_{i}(g)}}{|P|}+p^{2} R
$$

where the sum $\Sigma^{\prime}$ is over a set of class representatives $g$ and $R$ is some $p$-local rational number (coming from the contribution of $p$-regular elements). Multiplying this by $|P|$ we get

$$
|P|=\frac{\Lambda(1)^{2}}{|G: p|}+\Sigma^{\prime} \Lambda_{i}(g) \overline{\Lambda_{i}(g)}+p^{2}|P| R .
$$

Now, for $t=(|P|-1) / s$,

$$
\begin{aligned}
\sum^{\prime} \Lambda_{i}(g) \overline{\Lambda_{i}(g)} & =\Sigma^{\prime}\left(\delta \lambda_{i}(g)+c\right)\left(\delta \lambda_{i}(g)+c\right) \\
& =\sum^{\prime}\left(\lambda_{i}(g) \overline{\lambda_{i}(g)}+c \delta\left(\lambda_{i}(g)+\overline{\lambda_{i}(g)}\right)+c^{2}\right) \\
& =|P|-s-2 c \delta+c^{2} t .
\end{aligned}
$$

Since $|G: P| \equiv s(\bmod |P|), \Lambda(1) \equiv \delta s+c(\bmod |P|)$ and $p \mid \Lambda(1)$,

$$
\begin{aligned}
\frac{\Lambda(1)^{2}}{|G: P|} \equiv \frac{(\delta s+c)^{2}}{s}(\bmod p|P|) & \equiv \frac{s^{2}+2 \delta s c+c^{2}}{s} \\
& \equiv s+2 \delta c+\frac{c^{2}}{s} .
\end{aligned}
$$

Combining (1), (2) and (3) we find

$$
0 \equiv c^{2}(1+s t) \equiv c^{2}|P|(\bmod p|P|)
$$

This forces $p \mid c$. Since $p \mid \Lambda(1)$ we also have $p \mid(\delta s+c)$, whence $p \mid s$, a contradiction. This proves the Theorem.

Finally, we remark that the Theorem is probably true more generally. For instance, the situation where $P \in \operatorname{Syl}_{p}(G)$ and $C_{G}(x)=C_{G}(P)$ for all $x \in$ $P^{\#}$ could be tried. A proof similar to the above might work with the addition 
of some block calculations, as in [1, Part II].

\section{REFERENCES}

1. D. A. Sibley, Finite linear graps with a strongly self-centralizing Sylow subgroup. I, II, J. Algebra 36 (1975), 158-166; 319-332.

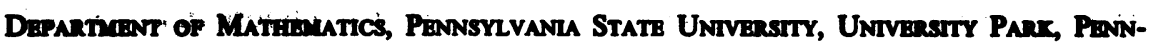
SYLVANI 16802 